\title{
Somatic/gonadal mosaicism for structural autosomal rearrangements: female predominance among carriers of gonadal mosaicism for unbalanced rearrangements
}

Natalia V. Kovaleva ${ }^{1 *}$ and Philip D. Cotter ${ }^{2,3}$

\begin{abstract}
Background: Mosaicism for chromosomal structural rearrangements (Rea) is rare and the timing and mechanisms of mosaic Rea formation, maintenance, and clinical manifestation are poorly understood. To date, there are no published data on the cytogenetic profile of mosaic Reas. The question as to whether the proportion of abnormal cells in the carrier's cultured blood is clinically significant remains unanswered. A previous study showed a strong female preponderance among carriers of mosaicism for Rea with pericentromeric breaks, indicating female-specific instability in early embryos. However, there is no corresponding study on male to female sex ratio (SR) among carriers of somatic and/or gonadal mosaicism for non-centromeric Rea. Population rates of mosaic Rea carriers calculated from consecutive series of patients referred for various reasons and from prenatal samples have not been established. Therefore the objectives of the present study were several-fold: (1) a study on profiles of Rea involved, (2) comparative analysis of the proportion of cells with unbalanced Rea in blood cultures from asymptomatic and affected carriers, (3) comparative analysis of SR in carriers of mosaicism for balanced and unbalanced Rea, and (4) determination of the population frequency of mosaicism for autosomal Rea.

(Continued on next page)
\end{abstract}

\footnotetext{
* Correspondence: kovalevanv2007@yandex.ru

'Department of Inherited Diseases, The Turner's Scientific and Research

Orthopaedic Institute for Children, Parkovaya Str. 64-68, St. Petersburg

196603, Russian Federation

Full list of author information is available at the end of the article
} 
(Continued from previous page)

Results: One hundred and three cases of mosaicism for autosomal non-centromeric Rea (N/Rea; normal line/ structural rearrangement) in which the sex of the carrier had been specified were identified in the literature. Among balanced Rea, there was a prevalence of reciprocal translocations (89\%) over inversions (11\%). Among unbalanced Rea, deletions were the most frequent (40\%), followed by duplications (25\%) and rings (16\%). Derivatives and other chromosome abnormalities were less frequent (9 and $10 \%)$. Eight of eleven (73\%) affected carriers of unbalanced Rea displayed a high proportion ( $>50 \%$ ) of abnormal cells compared to 4/37 (11 \%) in asymptomatic carriers, $p<0.0001$. Among carriers of mosaicism for balanced Rea there was a slight male predominance, $24 \mathrm{M} / 22 \mathrm{~F}$, unlike the strong female predominance among carriers of mosaicism for unbalanced Rea, $11 \mathrm{M} / 46 \mathrm{~F}, p<0.0001$. Among ten carriers of unbalanced Rea with reproductive failure, only one was a male with infertility, and one was a partner of a woman experiencing recurrent spontaneous abortion. Population rates of mosaics for reciprocal translocaton (N/rcp), inversion (N/inv), and unbalanced Rea (N/unbal Rea) calculated from published data on consecutive series of patients with reproductive failures were $0.02 \%$, $0.005 \%$, and $0.002 \%$, correspondingly. Among 30,376 infertile patients three carriers of mosaicism for balanced Rea were identified (two cases of N/rcp and one case of N/inv), whereas among 26,384 patients with habitual abortion seven carriers were detected (five N/rcp and two N/ inv). Among all 56,760 tested patients with reproductive failures only one was found to be a carrier of mosaicism for an unbalanced Rea (N/del, mosaicism for deletion).

Conclusions: A high proportion of Rea cells (>50\%) detected in cultured T-lymphocytes is associated with clinical manifestation of chromosomal imbalance. A strong female prevalence among carriers of mosaicism for unbalanced Rea suggests male-specific selection against abnormal cells rather than impairment of male gametogenesis, as the latter suggests a better prognosis for male fetuses. These findings should be taken into consideration when genetic counseling of patients referred after a diagnosis of mosaicism for an unbalanced rearrangement in a fetus.

\section{Background}

Mosaicism for structural chromosome abnormalities is rare and may be challenging for genetic counseling, particularly when detected prenatally. The identification and counseling of gonadal mosaicism (GM) may be even more problematic, being both asymptomatic and cryptic in the GM carrier.

There are two hypotheses for the existence of GM discussed in the literature. One is that the mutation occurs in a germ cell that continues to divide (mosaicism confined to germ cells). The other possibility is that the mutation occurs very early in a somatic cell before the separation to germinal cells and is therefore present both in somatic and germinal cells. Depending on various factors, such as the gene(s) involved and/or the degree of mosaicism, the carrier of a somatic and/or germline mosaicism may be asymptomatic (making GM difficult to detect) or may present with various symptoms of the disease [1]. A previous study reported a strong female preponderance among carriers of mosaicism for Rea with pericentromeric breaks indicating female-specific instability in early human embryos [2]. No data is available on the sex ratio among carriers of GM for non centromeric Rea.

The question as to whether the proportion of Rea cells in the carrier's cultured blood is clinically significant is unresolved. Data from Cheung et al. [3] confirmed the previous suggestion of Pagon et al. [4] that chromosome analysis of stimulated T-lymphocytes does not reflect the true rate of abnormal cells in a carrier of mosaicism. Considering that blood cultures (i.e. stimulated T-lymphocytes) are commonly employed in routine cytogenetic examination, it is of importance to study the problem thoroughly.

The objectives for this study were: (1) a study on profiles of Rea involved, (2) comparative analysis of the proportion of cells with unbalanced Rea in blood cultures from asymptomatic and affected carriers, (3) comparative analysis of SR in carriers of mosaicism for balanced and unbalanced Reas, and (4) determination of the population frequency of mosaicism for autosomal Rea.

\section{Materials and methods}

We reviewed reports in the literature of mosaicism for $\mathrm{N} /$ Rea cases detectable microscopically (up to 850-band level of resolution) either by conventional cytogenetics or by molecular cytogenetics. The cases were identified from various sources including PubMed using combinations of the search terms "mosaicism", "mosaic", "recurrent", "inherited", "familial", 'transmitted", "maternal, "paternal", and "parental". Only reports of N/Rea carriers of known sex were selected for the study. From the sample collected we excluded cases of Rea with both breakpoints localized at pericentromeric regions, because of the strong female preponderance among carriers of such mosaicism [2, 5]. Cases of Rea transmitted from a carrier parent rescued along with the formation a normal line were similarily not included in the study. The majority of the cases reported 
since 2000 were detected, verified and/or analysed using molecular cytogenetic and molecular technologies.

One hundred and four cases of carriers of N/Rea, along with the data on their chromosome constitution, carrier's age at the birth of the proband (when relevant and/or specified), proportion of abnormal cell line(s), and the indication for testing have been identified and subdivided as follows: affected carriers of apparent GM with abnormal offspring, asymptomatic carriers of GM with abnormal offspring, asymptomatic carriers of GM with healthy offspring, asymptomatic carriers of somatic mosaicism (SM) assumed to have GM, i.e. patients with poor reproductive history, and asymptomatic carriers of somatic mosaicism fortuitously detected. According to Barber [6] (the majority of the cases of affected carriers with abnormal offspring in the present study were retrieved from this review), individuals were considered phenotypically affected when any type of phenotypic anomaly was reported even if the etiological role of the chromosome abnormality in the same individual is questionable. We also analyzed available published data on consecutive series of patients/couples experiencing reproductive problems, aiming to estimate a population frequency of N/Rea mosaicism. Data were analyzed using standard statistics, a Chi-square test with Yates correction. The comparison of observed and expected proportions was made using binomial test.

\section{Results and discussion}

\section{N/Rea profile in studied groups}

There is a prominent difference between studied groups regarding the proportion and profiles of unbalanced and balanced Rea. As seen from Table 1, among 12 affected N/ Rea carriers with affected offspring who inherited the same non mosaic Rea, there were no carriers of balanced translocations. Among them, carriers of deletions (including ring chromosomes) and duplications were represented equally.

Among asymptomatic carriers with affected offspring (Table 2), 33 of 42 were mosaics for an unbalanced Rea, with some prevalence of deletions (16 cases including ring chromosomes) over duplications (8 cases). Additionally, there were four cases of unbalanced translocation and five cases of other Rea. Among mosaics for balanced Rea, two were carriers of an inversion, six were carriers of a reciprocal translocation, and one was a carrier of insertion.

A different proportion between unbalanced and balanced Rea was observed in the remaining three groups of patients with SM/GM. Asymptomatic carriers with healthy offspring carrying the same Rea (Table 3), showed a substantial prevalence of balanced Rea (8 of 10 cases). In the group of asymptomatic carriers with poor reproduction (Table 4) balanced Rea also prevailed over unbalanced Rea (23 cases vs 10 cases). Among the latter group there was a significant predominance of deletions including rings over duplications (9 cases vs 1 case). Finally, among asymptomatic carriers of SM detected fortuitously (Table 5), we detected six carriers of a balanced Rea.

Overall, among balanced Rea, there was a prevalence of reciprocal translocations over inversions (89 and $11 \%$ ). As to the distribution of unbalanced Rea, deletions were the most frequent (40\%), followed by duplications ( $24 \%$ ), and rings (17\%). Derivatives and other chromosome abnormalities were less frequent ( 9 and $10 \%$ ).

A low proportion of mosaics for derivative chromosome can readily be explained by the mechanism of their formation, i.e. postzygotic non-homologous recombination or nonhomologous end-joining [7].

\section{Proportion of cells with unbalanced Rea in blood cultures from asymptomatic and affected carriers}

The proportion of abnormal cells was reported in 89 cases. On average, in asymptomatic carriers of a balanced Rea $(n=41)$, the mean proportion of abnormal cells was $33 \%$, and the corresponding figure for asymptomatic carriers of unbalanced Rea $(n=38)$ was $20 \%$. In contrast, the mean proportion of abnormal cells in affected carriers of unbalanced Rea $(n=11)$ was $63 \%$. Since the number of tested cells was not specified in every case, a valid statistical analysis of the figures obtained was not possible.

Therefore, we analyzed a number of individuals with a proportion of abnormal cells reported to be larger than $50 \%$. A remarkable difference was found between asymptomatic carriers of unbalanced Rea and affected carriers of unbalanced Rea: 4 of $37(11 \%)$ vs 8 of 11 (73\%), $p<0.0001$. Unfortunately because of few reports of such cases, the size of the latter group is small.

The reliability of routine chromosome analysis of stimulated T-lymphocytes from blood for detection and evaluation of mosaicism was questioned when higher rates of detection of mosaicism in cultured skin fibroblasts became evident [8-10]. Recent studies using array CGH confirmed that conventional cytogenetic methods underestimate the level of mosaicism [3]. However, although undoubtedly array $\mathrm{CGH}$ and single-nucleotide polymorphism (SNP) microarrays are superior to other methodologies in detecting somatic chromosome mosaicism [3, 11], it should be acknowledged that currently conventional chromosome analysis is the most readily available method worldwide and will be so in the foreseeable future. Therefore, awareness of an association of a high rate of abnormal cells in cultured T-lymphocytes with clinical manifestation of chromosomal imbalance might be helpful, particularly if this is confirmed in studies on prenatal cases.

Nevertheless, it should be noted that when GM is suspected in the absence of SM in blood cultures, further 
Table 1 Saawomatic/gonadal mosaicism for non centromeric rearrangement in affected carriers with affected offspring

\begin{tabular}{|c|c|c|c|c|}
\hline Reference & Karyotype & $\begin{array}{l}\text { Age at birth } \\
\text { of the } \\
\text { proband }\end{array}$ & $\begin{array}{l}\text { Proportion of } \\
\text { abnormal cell } \\
\text { line(s) }\end{array}$ & Indication for testing \\
\hline \multicolumn{5}{|c|}{ Unbalanced rearrangements } \\
\hline \multicolumn{5}{|l|}{ N/del } \\
\hline $\begin{array}{l}\text { Freitas et al., } \\
2012[25]\end{array}$ & 46,XX/46,XX,del(2)(q36.1q36.3) & $23 \mathrm{yr}$ & $\begin{array}{l}90 \% \mathrm{BL}, 10 \% \\
\text { DNA }\end{array}$ & mild presentation of MFDH; a child with the same Rea \\
\hline $\begin{array}{l}\text { Naritomi, Hirayama, } \\
1989 \text { [26] }\end{array}$ & $46, X X / 46, X X$,del(8)(q23.3q24.13) & $34 \mathrm{yr}$ & $50 \%$ & $\begin{array}{l}\text { mild trichorhoniphalangeal syndrome I in the mother, } \\
\text { a child with the same Rea }\end{array}$ \\
\hline $\begin{array}{l}\text { Magenis et al., } \\
1989 \text { [27] }\end{array}$ & $46, X X / 46, X X$,del(9q31.3) & ns & ns & $\begin{array}{l}\text { mild mental retardation, affected child with the } \\
\text { same Rea }\end{array}$ \\
\hline $\begin{array}{l}\text { Zori et al., } 1993 \\
\text { [28] (patient B) }\end{array}$ & 46,XX/46,XX,del(17)(p11.2p12) & $30 \mathrm{yr}$ & $55 \% \mathrm{BL}$ & partially affected, a child with SMS \\
\hline \multicolumn{5}{|l|}{ N/dup } \\
\hline $\begin{array}{l}\text { Cox et al., } 2002 \\
\text { [29] }\end{array}$ & 46,XX/46,XX,dup(7)(?p15.3?p22) & $40 \mathrm{yr}$ & $83 \% \mathrm{BL}$ & $\begin{array}{l}\text { global intellectual impairment, rebellious behavior, } \\
\text { craniofacial dysmorphism, a child with the same Rea }\end{array}$ \\
\hline $\begin{array}{l}\text { Kennedy et al., } \\
2001[30]\end{array}$ & 46,XY/46,XY, dup(8)(p23.1p23.1) & ns & $68 \% \mathrm{BL}$ & congenital heart defect, a child with the same Rea \\
\hline $\begin{array}{l}\text { Pfeiffer and } \\
\text { Schutz, } 1993 \text { [31] }\end{array}$ & $46, X X / 46, X X$,dir dup(11)(q23->qter) & $26 \mathrm{yr}$ & $19 \% B L$ & $\begin{array}{l}\text { mildly retarded; a dysmorphic child with the same } \\
\text { Rea }\end{array}$ \\
\hline $\begin{array}{l}\text { Barber et al., } \\
2006 \text { [32] (family } \\
\text { 1) }\end{array}$ & $\begin{array}{l}46, X X / 46, X X, \text { inv dup } \\
\text { ins(16)(q11.2q13q11.2) }\end{array}$ & $33 \mathrm{yr}$ & $52 \% \mathrm{BL}$ & $\begin{array}{l}\text { developmental delay and phenotypic abnormality, } \\
\text { affected child with the same Rea }\end{array}$ \\
\hline $\begin{array}{l}\text { Moog et al., } 1994 \\
\text { [33] (patient B) }\end{array}$ & 46,XX/46,XX,dup18(pter->cen) & $26 \mathrm{yr}$ & $80 \%$ BL & dysmorphic, slightly mentally retarded, son with dup(8) \\
\hline \multicolumn{5}{|l|}{ N/ring } \\
\hline $\begin{array}{l}\text { Fryns, Van den } \\
\text { Berghe, } 1979[34]\end{array}$ & $46, X X / 46, X X, r(22) / 45, X X, t(15 q 21 q)$ & $21 \mathrm{yr}$ & $\begin{array}{l}32 \% / 65 \% \mathrm{BL}, \\
19 \% / 24 \% \mathrm{SF}\end{array}$ & slightly mentally retarded, a child with the same Rea \\
\hline \multicolumn{5}{|l|}{$\mathrm{N} / \mathrm{t}$ unbalanced } \\
\hline $\begin{array}{l}\text { De Pater et al., } \\
2003 \text { [35] }\end{array}$ & $\begin{array}{l}46, X X, \operatorname{der}(18) t(18 ; 21)(q 21.3 ; p 12) / \\
46, X X, \operatorname{der}(21) t(18 ; 21)(q 21.3 ; p 12){ }^{a}\end{array}$ & $37 \mathrm{yr}$ & $47 \% / 53 \% \mathrm{BL}$ & $\begin{array}{l}\text { very mild phenotypic abnormalities, a child with } \\
18 \mathrm{q} \text { - syndrome }\end{array}$ \\
\hline \multicolumn{5}{|l|}{ Nother rea } \\
\hline $\begin{array}{l}\text { Galjaard et al., } \\
2003[36]\end{array}$ & $\begin{array}{l}46, X Y / 46, X Y, t(4 ; 7)(p 15.2 ; q 35) \\
\text { microdeletions at both } \operatorname{der}(4) \text { and } \\
\operatorname{der}(7)\end{array}$ & ns & $70 \% \mathrm{BL}, 96 \% \mathrm{SF}$ & $\begin{array}{l}\text { isolated postaxial polydactyly, affected child with the } \\
\text { same Rea }\end{array}$ \\
\hline Total & 2 males 10 females & & & \\
\hline
\end{tabular}

${ }^{a}$ presence of normal cell line can be suggested confidently because of very mild clinical manifestation; healthy $46, X Y$ child BL, blood culture (i.e. stimulated T-lymphocytes)

$\mathrm{SF}$, skin fibroblasts culture

application of modern technologies is desirable, either for searching for the abnormal cell line(s) in different tissues or for identification of the parental origin of the recurrent Rea detected in the offspring.

Sex ratio in carriers of GM for balanced and unbalanced Reas As summarized in Table 6, among affected carriers of GM, there is a notable female predominance (2 M/10 F). Among asymptomatic carriers of unbalanced Rea, both carriers of GM and carriers of SM, there is also a significant prevalence of females $(9 \mathrm{M} / 38 \mathrm{~F}$ and $2 \mathrm{M} / 8 \mathrm{~F})$. In contrast, both asymptomatic carriers of proven GM and asymptomatic carriers of SM for balanced Rea show a slight, but not significant, prevalence of males $(9 \mathrm{M} / 8 \mathrm{~F}$ and $15 \mathrm{M} / 14 \mathrm{~F}$, correspondingly). Overall, carriers of unbalanced Rea demonstrate a highly significant fiour-fold female predominance $(11 \mathrm{M} / 46 \mathrm{~F}, \mathrm{SR}=0.24)$, different from population ratio of 1.06 at $p<0.0001$, while male predominance $(\mathrm{SR}=1.09)$ among carriers of mosaicism for balanced Rea is not different from population ratio of 1.06 .

Considerable, but not several-fold, prevalence of females over males among carriers of non-mosaic reciprocal translocations, both referred for prenatal testing for the presence of chromosomal Rea and those diagnosed as Rea carriers during prenatal testing, is well documented [12-14]. A similar female predominance was found among carriers of reciprocal translocations experiencing repeated miscarriages (see Table 7). This 
Table 2 Somatic/gonadal mosaicism for non centromeric rearrangement in asymptomatic carriers with affected offspring

\begin{tabular}{|c|c|c|c|c|}
\hline Reference & Karyotype & $\begin{array}{l}\text { Carrier's age at birth } \\
\text { of the proband }\end{array}$ & $\begin{array}{l}\text { Proportion of abnormal } \\
\text { cell line(s) }\end{array}$ & Indication for testing \\
\hline \multicolumn{5}{|c|}{ Unbalanced rearrangements } \\
\hline \multicolumn{5}{|l|}{$\mathrm{N} / \mathrm{del}$} \\
\hline $\begin{array}{l}\text { Galan-Gomez et al., } \\
1994 \text { [37] }\end{array}$ & 46,XX/46,XX,del(5)(p14-pter) & ns & ns & children with $5 p$ - syndrome \\
\hline $\begin{array}{l}\text { Johnson et al., } \\
2000 \text { [38] }\end{array}$ & $46, X Y / 46, X Y$, del $(5)(p 14)$ & ns & $100 \% \mathrm{BL}, 99 \% \mathrm{SF}$ & $\begin{array}{l}\text { affected child with the same } \\
\text { deletion }\end{array}$ \\
\hline $\begin{array}{l}\text { McDonald et al., } \\
1988 \text { [39] (family 3) }\end{array}$ & $46, X X / 46, X X$,del(5)(p14) & ns & ns & a child with del(5) \\
\hline Niebuhr, 1978 [40] & $46, X X / 46, X X$,del(5)(p14) & ns & $5 \% \mathrm{BL}, 3 \% \mathrm{SF}$ & a child and fetus with the same Rea \\
\hline $\begin{array}{l}\text { Van Tuinen et al., } \\
2001 \text { [41] }\end{array}$ & 46,XX/46,XX,del(5)(p14.2) & ns & $4 \% \mathrm{BL}$ & $\begin{array}{l}\text { a child with cri-du-chat } \\
\text { syndrome }\end{array}$ \\
\hline $\begin{array}{l}\text { Brandriff et al., } \\
1988 \text { [42] }\end{array}$ & 46, XX/46,XX,del(13)(q22q32) & ns & $0 \% \mathrm{BL}, 0 \% \mathrm{SF}$ a) & $\begin{array}{l}\text { a chid and fetus with the same } \\
\text { Rea }\end{array}$ \\
\hline $\begin{array}{l}\text { Michalova et al., } \\
1982 \text { [43] }\end{array}$ & $46, X X / 46, X X$,del(13)(q12->q31) & $18 \mathrm{yr}$ & $3 \% \mathrm{BL}$ & child with retinoblastoma \\
\hline $\begin{array}{l}\text { Kokkonen, Leisti, } \\
2000[44]\end{array}$ & $46, X X / 46, X X$, del(15)(q1 1q13) & ns & $0 \% \mathrm{BL} b)$ & two affected children \\
\hline $\begin{array}{l}\text { Rump et al., } \\
2008 \text { [45] }\end{array}$ & 46,XX/46,XX,del(15)(q26.2->qter) & ns & $0 \% \mathrm{BL} b)$ & two children with del(15) \\
\hline $\begin{array}{l}\text { Sanchez et al., } \\
2014[46]\end{array}$ & $46, X X / 46, X X$, del(15)(q11.2q13) & ns & $\begin{array}{l}\text { 0\% BL, } 0 \% \text { normal SF, } \\
35 \% \text { hypopigmented SF }\end{array}$ & $\begin{array}{l}\text { dyzygotic twins with Angelman } \\
\text { syndrome }\end{array}$ \\
\hline $\begin{array}{l}\text { Hoo et al., } \\
1985 \text { [47] }\end{array}$ & $46, X X / 46, X X$,del(16)(q11.1q12.1) & $22 \mathrm{yr}$ & $0 \% \mathrm{BL}, 0 \% \mathrm{SF}$ c) & two children with del(16) \\
\hline $\begin{array}{l}\text { Garcia-Heras et al., } \\
2005 \text { [48] }\end{array}$ & 46,XX/46,XX,del(20)(p11.1p12) & $33 \mathrm{yr}$ & $25 \% B L$ & child with the same deletion \\
\hline \multicolumn{5}{|l|}{ N/dup } \\
\hline $\begin{array}{l}\text { Eussen et al., } \\
2007 \text { [49] }\end{array}$ & 46,XX/46,XX,inv dup(2)(q34q33) & $28 \mathrm{yr}$ & $19 \% B L$ & two children with the same Rea \\
\hline $\begin{array}{l}\text { Bernardini et al., } \\
2005[50]\end{array}$ & 46,XX/46,XX,dup(4)(p15p15) & young & $\begin{array}{l}30 \% \mathrm{BL}, 20-25 \% \\
\text { different tissues }\end{array}$ & three abortions with the same Rea \\
\hline $\begin{array}{l}\text { Toska et al., } \\
2010 \text { [51] }\end{array}$ & $46, X X / 46, X X, \operatorname{dup}(4)(q 22.2 q 23)$ & $24 \mathrm{yr}$ & $0 \% B L b)^{*}$ & two siblings with dup(4) \\
\hline $\begin{array}{l}\text { Fan et al., } 2001 \\
\text { [52] (family 2) }\end{array}$ & 46,XY/46,XY,dup(8)(p21.3p23.1) & ns & $20 \% B L$ & two children with the same Rea \\
\hline $\begin{array}{l}\text { Tonk et al., } \\
1996[53]\end{array}$ & $46, X X / 46, X X$,dir dup(10)(q24.2->q24.3) & ns & $10 \%$ & two children with dup(10) \\
\hline $\begin{array}{l}\text { Hocking et al., } \\
1999[54]\end{array}$ & 46,XY/46,XY,dup(13)(q32q34) & ns & ns & a child with the same Rea \\
\hline $\begin{array}{l}\text { Babovic-Vuksanovic } \\
\text { et al., } 1998 \text { [55] }\end{array}$ & $46, X X / 46, X X, \operatorname{dup}(17)(q 24 q 25.1)$ & $16 \mathrm{yr}$ & $29 \%$ BL & $\begin{array}{l}\text { recurrent abortins, two children } \\
\text { with dup(17) }\end{array}$ \\
\hline $\begin{array}{l}\text { Flowers et al., } \\
2015 \text { [56] }\end{array}$ & $46, X X / 46, X X, \operatorname{dup}(18)(q 12.1 q 21.1)$ & $38 \mathrm{yr}$ & $20 \% B L$ & a fetus with the same Rea \\
\hline \multicolumn{5}{|l|}{ N/ring } \\
\hline $\begin{array}{l}\text { Meza-Espinoza } \\
\text { et al., } 2008 \text { [57] }\end{array}$ & $46, X Y / 46, X Y, r(17)$ & ns & $4 \% \mathrm{BL}$ & $\begin{array}{l}\text { a child with multiple anomalies } \\
\text { with the same Rea }\end{array}$ \\
\hline $\begin{array}{l}\text { Fryns et al., } \\
1992[58]\end{array}$ & $46, X X / 46, X X, r(18)(p 11.3 q 23)$ & $26 \mathrm{yr}$ & $8 \% B L$ & polymalformed child with $r(18)$ \\
\hline $\begin{array}{l}\text { Flejter et al., } \\
1996[59]\end{array}$ & $46, X X / 46, X X, r(19)$ & $27 \mathrm{yr}$ & $4 \% B L$ & affected child with the same Rea \\
\hline Phelan, & $46, X X / 46, X X, r(22)$ & ns & ns & affected child with the same Rea \\
\hline
\end{tabular}


Table 2 Somatic/gonadal mosaicism for non centromeric rearrangement in asymptomatic carriers with affected offspring (Continued)

\begin{tabular}{|c|c|c|c|c|}
\hline \multicolumn{5}{|l|}{$\mathrm{N} / \mathrm{t}$ unbalanced } \\
\hline $\begin{array}{l}\text { Engel et al., } \\
2001[61]\end{array}$ & 46,XX/46,XX,psudic(5;21)(q12.p13) & young & $0 \% \mathrm{BL}, 0 \% \mathrm{SF}$ b) & two children with psudic $(5 ; 21)$ \\
\hline $\begin{array}{l}\text { Kouru et al., } \\
2011[62]\end{array}$ & 46,XX/45,XX,psu dic(5;22)(p15.p11.1) & ns & $0 \% \mathrm{BL}, 5 \% \mathrm{SF}$ & $\begin{array}{l}\text { a child and a fetus with the } \\
\text { same Rea }\end{array}$ \\
\hline $\begin{array}{l}\text { Gijsbers et al., } \\
2011 \text { [63] (case 2) }\end{array}$ & $46, X X / 46, X X, \operatorname{der}(22) t(8 ; 22)(q 24.2 ; p 10)$ & ns & $52 \% \mathrm{BL}$ & $\begin{array}{l}\text { affected daughter with the } \\
\text { same Rea }\end{array}$ \\
\hline $\begin{array}{l}\text { Papenhausen et al., } \\
1991 \text { [64] }\end{array}$ & $46, X X / 46, X X, \operatorname{der}(21) t(21 ; 21 ;)(p 11 ; q 22.1)$ & $29 \mathrm{yr}$ & $30 \% \mathrm{BL}$ & a child with the same Rea \\
\hline \multicolumn{5}{|l|}{ N/other rea } \\
\hline $\begin{array}{l}\text { Al Arrayed, } 1998 \\
\text { [65] (case 10) }\end{array}$ & $46, X Y / 46, X Y$,multiple rea(2) & ns & ns & three abnormal children \\
\hline $\begin{array}{l}\text { Eckel et al., } \\
2006 \text { [66] }\end{array}$ & $\begin{array}{l}\text { 46,XX/46,XX,trp(12)(pter->p11.22-> } \\
\text { p12.3:"p12.3->qter) }\end{array}$ & ns & $12 \% B L$ & affected child with the same Rea \\
\hline $\begin{array}{l}\text { Masada et al., } \\
1989[67]\end{array}$ & $\begin{array}{l}\text { 46,XY/46,XY, del(14)(q32.11->qter)/ } \\
\text { 46,XY,dup(14)(q32.11->qter) }\end{array}$ & ns (mother $31 \mathrm{yr}$ ) & $0 \% \mathrm{BL}, 0 \% \mathrm{SF} d)$ & $\begin{array}{l}\text { a child with del(14), a child with } \\
\text { dup(14) }\end{array}$ \\
\hline $\begin{array}{l}\text { Insley et al., } \\
1968 \text { [68] }\end{array}$ & $46, X X / 46, X X, D q+$ & $22 \mathrm{yr}$ & $2 \% \mathrm{BL}, 3 \% \mathrm{SF}$ & two daughters with the same Rea \\
\hline $\begin{array}{l}\text { D'Angelo et al., } \\
2010 \text { [69] }\end{array}$ & $\begin{array}{l}\text { 46,XX/ } \\
46, X X \text {,del(20)(p11.21)dup(20)(p11.21.p13) }\end{array}$ & $23 \mathrm{yr}$ & $15 \% B L$ & $\begin{array}{l}\text { affected daughter with the } \\
\text { same Rea }\end{array}$ \\
\hline Total & 6 males 27 females & & & \\
\hline \multicolumn{5}{|c|}{ Balanced rearrangements } \\
\hline \multicolumn{5}{|l|}{ N/inv } \\
\hline $\begin{array}{l}\text { Shapira et al., } \\
1997 \text { [22] }\end{array}$ & 46,XY/46,XY,inv(9)(p24q34.1) & $25 \mathrm{yr}$ & $25 \% B L$ & $\begin{array}{l}\text { a child with recombinant } 9 p \\
\text { aneusomy }\end{array}$ \\
\hline $\begin{array}{l}\text { Wang et al., } \\
2010[70]\end{array}$ & $46, X Y / 46, X Y, \operatorname{inv}(20)(p 12.2 q 13.33)$ & $35 \mathrm{yr}$ & $50 \% \mathrm{BL}$ & $\begin{array}{l}\text { two children with recombinant } \\
\text { chromosome } 20\end{array}$ \\
\hline \multicolumn{5}{|l|}{$\mathrm{N} / \mathrm{t}$ balanced } \\
\hline $\begin{array}{l}\text { Aurias et al., } \\
1978[71]\end{array}$ & $46, X X / 46 / X X, t(2 ; 4)(q 37 ; q 28)$ & ns & ns & a child with der(4) t(2;4)(q37;q28) \\
\hline $\begin{array}{l}\text { Becker, Albert, } \\
1963 \text { [72] }\end{array}$ & $46, X Y / 45, X Y$, nonacrocentric $t(2 ; 21)$ & 23 & $55 \% \mathrm{BL}$ & $\begin{array}{l}\text { neurofibromatosis, a child with } \\
\text { Down syndrome }\end{array}$ \\
\hline $\begin{array}{l}\text { Gardner et al., } \\
1994 \text { [73] (case 7) }\end{array}$ & $46, X X / 46, X X, r c p(5 ; 18)(p 15 ; q 21)$ & ns & $0,1 \% \mathrm{BL}, 0 \% \mathrm{SF}$ & a child with der (18) \\
\hline $\begin{array}{l}\text { Simonova et al., } \\
2005 \text { [74] }\end{array}$ & $46, X Y / 46, X Y, t(5 ; 20)(p 12 ; q 13)$ & ns & $8 \% \mathrm{BL}$ & a child with del(5) \\
\hline $\begin{array}{l}\text { Sciorra et al., } \\
1992[75]\end{array}$ & $46, \mathrm{XY} / 46, \mathrm{XY}, \mathrm{t}(7 ; 14)(\mathrm{q} 36 ; \mathrm{q} 1 ?)$ & ns (mother $31 \mathrm{yr}$ ) & $0.5 \% \mathrm{BL}, 0 \% \mathrm{SF}$ & a child with $7 q+$ \\
\hline $\begin{array}{l}\text { Opheim et al., } \\
1995 \text { [76] (case 2) }\end{array}$ & $46, X X / 46, X X, t(8 ; 13)(p 23.2 ; q 21.2)$ & ns & $57 \% \mathrm{BL}$ & a child with $\operatorname{der}(8) t(8 ; 13)$ \\
\hline $\begin{array}{l}\text { Yatsenko et al., } \\
2009[77]\end{array}$ & $46, X X / 46, X X$, ins (12)(q12p11.1p13.1) & ns & $50 \% \mathrm{BL}$ & $\begin{array}{l}\text { two children with Noonan } \\
\text { syndrome and the same Rea }\end{array}$ \\
\hline Total & 5 males 4 females & & & \\
\hline
\end{tabular}

a) ovarian germinal mosaicism deduced from absence of the Rea in sperm chromosomes

b) ovarian germinal mosaicism deduced from molecular analysis

c) maternal origin proved by $16 \mathrm{qh}$ heteromorphism

d) paternal origin proved by $14 \mathrm{p}$ heteromorphism

has been commonly explained by male sterility [15-18]. However analysis of the literature (Table 7) did not show a correspondingly significant predominance of males over female among infertile carriers of reciprocal translocation $(\mathrm{SR}=1.2$, not significantly different from 1.06). Moreover, the rate of reciprocal translocations in infertile males is even lower compared to the reciprocal translocations rate in males from couples experiencing repeated miscarriage $(0.53 \%$ vs $0.86 \%)$. 
Table 3 Somatic/gonadal mosaicism for non centromeric rearrangement in asymptomatic carriers with unaffected offspring

\begin{tabular}{|c|c|c|c|c|}
\hline Reference & Karyotype & $\begin{array}{l}\text { Age at } \\
\text { ascertainment }\end{array}$ & $\begin{array}{l}\text { Proportion of abnormal } \\
\text { cell line(s) }\end{array}$ & Indication for testing \\
\hline \multicolumn{5}{|l|}{ Unbalanced rearrangements } \\
\hline Tinkel-Vernon et al., 2001 [78] & $46, X Y / 46, X Y$,del(21)(q11.2->q21) & ns & $50 \% \mathrm{BL}$ & normal son with the same Rea \\
\hline Mazzaschi et al., 2011 [79] & $46, X X / 46, X X, r(21)$ & $40 \mathrm{yr}$ & $30 \% \mathrm{BL}$ & normal child with the same Rea \\
\hline Total & 1 male 1 female & & & \\
\hline \multicolumn{5}{|l|}{ Balanced rearrangements } \\
\hline Kleczkowska et al., 1990 [21] (case 1) & $46, X Y / 46, X Y, t(1 ; 9)(p 13.1 ; p 12.2)$ & ns & $50 \% \mathrm{BL}$ & Two healthy children the same Rea \\
\hline Zackovsi et al., 1995 [80] & 46,XY/46,XY,inv(1)(p31.2p34.3) & ns & $28 \% \mathrm{BL}$ & normal child with inv(1) \\
\hline Heil et al., 1997 [81] (case 1) & $46, X Y / 46, X Y, t(2 ; 3)(q 37 ; q 21)$ & ns & $19 \% \mathrm{BL}$ & a fetus with $t(2 ; 3)$ \\
\hline Farrell, 1991 [82] (case 2) & $46, X X / 46, X X, r c p(5 ; 18)(q 35 ; q 21.3)$ & ns & $28 \% \mathrm{BL}$ & $\begin{array}{l}46, X Y, t(5 ; 18) \text { son with reproduction } \\
\text { failure }\end{array}$ \\
\hline Leegte et al., 1998 [83] (case 1) & $46, X X / 46, X X, t(9 ; 15)(q 12 ; p 11.2)$ & ns & $32 \% \mathrm{BL}$ & $46, X Y, t(9 ; 15)$ son with infertility \\
\hline Dupont et al., 2008 [84] & $46, X X / 46, X X, t(9 ; 22)(q 34.3 ; q 13.3)$ & ns & $50 \% \mathrm{BL}$ & $\begin{array}{l}\text { affected grandchild with } 46, X Y \\
\text { der(22)t( }(9 ; 22) \text { (q34.3;q13.3) }\end{array}$ \\
\hline Storto et al., 1999 [85] & $46, X Y / 46, X Y, 10 q s$ & ns & $60 \% \mathrm{BL}$ & normal child with 10qs \\
\hline Gardner et al., 1994 [73] (case 12) & $46, X X / 46, X X, r c p(11 ; 22)(q 23 ; q 21)$ & ns & $62 \% \mathrm{BL}$ & a grandchild with $\operatorname{der}(22) t(11 ; 22)$ \\
\hline Total & 4 males 4 females & & & \\
\hline
\end{tabular}

While one might expect a female predominance among asymptomatic carriers of GM for balanced Rea (mostly reciprocal translocations), who were diagnosed as such because of their abnormal offspring, this was not observed. However, among carriers of GM for unbalanced Rea there was a strong female prevalence. The same profile was found in the subgroup of carriers of SM mosaicism.

Mosaicism for unbalanced Rea does not appear to be a significant reason for male sterility, since among ten asymptomatic carriers with reproductive failure (Table 4) only one was a male with infertility, and another was a partner of a woman experiencing recurrent spontaneous abortion. Moreover, as seen from Table 7, among 200 infertile males diagnosed as carriers of a chromosome abnormality, none were diagnosed as a carrier of SM for unbalanced Rea. Consequently, other mechanism(s) resulting in the strong female predominance among carriers of mosaicism for unbalanced Rea can be postulated, including a high intrauterine lethality of male carriers, a male-specific selection against abnormal cells in the early embryo development, or a high instability in the early female embryo development.

A high intrauterine lethality of male carriers can be excluded because of significant predominance of females among abortuses with mosaicism for unbalanced Rea (Kovaleva, unpublished). Male-specific selection against abnormal cells in early embryo development seems more plausible. Several authors suggested that female embryos are relatively delayed in early embryonic development $[19,20]$. The delay in early female development has been ascribed to the absence of a Y chromosome.
However, the process of $\mathrm{X}$ inactivation, since it may occur when there are $\leq 10$ cells in the embryo might itself contribute to a slight delay in early female embryo development [19]. A higher male cell turnover might facilitate effective selection against abnormal cell line.

High instability in early female embryo development would predict a female prevalence would be expected among both carriers of balanced and unbalanced Rea, arguing against this mechanism. However, the strong female prevalence is only observed among carriers of unbalanced Rea. Additional studies of the phenomenon of multifold female predominance among carriers of somatic and/or gonadal mosaicism for unbalanced Rea will add a new dimension to diversity of manifestation of human sexual dimorphism.

\section{Estimation of detection frequency of somatic N/Rea mosaicism}

The results of the combined data on structural autosomal Reas excluding supernumerary marker chromosomes (SCM) detected in 56,760 patients referred for chromosome testing for reproductive failure are presented in Table VII. Among them, 833(1.46 \%) individuals were found to be carriers of structural chromosomal non mosaic abnormalities, and 13 (0.02 \%) were carriers of N/Rea. Among balanced Reas, mosaics for inversions were the most frequent $(3 / 103=2.9 \%)$, the reciprocal translocations $(7 / 453=1.5 \%)$, while mosaics for Robertsonian translocation were less frequent $(2 / 265=0.8 \%)$, A majority of mosaics (10/11) were balanced Reas, and only one of 56,760 tested patients 
Table 4 Somatic mosaicism for non centromeric rearrangement in asymptomatic carriers with poor reproductive history

\begin{tabular}{|c|c|c|c|c|}
\hline Reference & Karyotype & $\begin{array}{l}\text { Age at } \\
\text { ascertainment }\end{array}$ & $\begin{array}{l}\text { Proportion of } \\
\text { abnormal cell line(s) }\end{array}$ & Indication for testing \\
\hline \multicolumn{5}{|c|}{ Unbalanced rearrangements } \\
\hline \multicolumn{5}{|l|}{ N/del } \\
\hline $\begin{array}{l}\text { D'Alessandro } \\
\text { et al., } 1992 \text { [86] }\end{array}$ & 46,XX/46,XX,del(6)(p23) & ns & $11 \% \mathrm{BL}$ & $\begin{array}{l}\text { recurrent abortions and idiopathic } \\
\text { hyporpolactinemia }\end{array}$ \\
\hline $\begin{array}{l}\text { Reddy, } 1999 \\
\text { (case 3) [87] }\end{array}$ & 46,XX/46,XX,del(8)(p23.1) & $29 \mathrm{yr}$ & $22 \% \mathrm{BL}$ & recurrent SA \\
\hline $\begin{array}{l}\text { Kleczkowska, } \\
\text { Fryns, } 1990[88]\end{array}$ & $\begin{array}{l}46, X X / 46, X X \text {,del(11)(:q14.2- } \\
>\text { q23.2:) }\end{array}$ & $28 \mathrm{yr}$ & $25 \% \mathrm{BL}$ & recurrent SA \\
\hline $\begin{array}{l}\text { Dutta et al., } \\
2011 \text { [89] }\end{array}$ & $46, X X / 46, X X$,del(17)(q) & ns & ns & recurrent miscarriage \\
\hline $\begin{array}{l}\text { Sachs et al., } \\
1985 \text { [90] }\end{array}$ & 46,XX/46,XX,del(20)(p12) & ns & $22 \%$ & recurrent SA \\
\hline \multicolumn{5}{|l|}{ N/dup } \\
\hline $\begin{array}{l}\text { Somprasit et al. } \\
2004 \text { [91] }\end{array}$ & 46,XY/46,XY,dup(21q22.13-q22.2) & $33 \mathrm{yr}$ & $0 \% \mathrm{BL}, 7 \%$ sperm & recurrent SA \\
\hline \multicolumn{5}{|l|}{ N/ring } \\
\hline Lee, 2002 [92] & $\begin{array}{l}46, X X / 46, X X, r(4) / 45, X X,-4 / 46, X X, \text { dic } \\
r(4) / 47, X X, r(4),+r(4)\end{array}$ & $27 \mathrm{yr}$ & $75 \% / 8 \% / 5 \% / 4 \%$ & infertility and short stature \\
\hline $\begin{array}{l}\text { Scholtes et al., } \\
1998 \text { [93] }\end{array}$ & $46, X X / 46, X X, r(14)$ & ns & ns & infertility, pre-ICSI testing \\
\hline $\begin{array}{l}\text { Tarlatzis et al., } \\
2000 \text { [94] }\end{array}$ & $46, X X / 46, X X, r(14)$ & ns & ns & infertility \\
\hline $\begin{array}{l}\text { Hammoud et al., } 2009 \\
\text { [95] }\end{array}$ & $46, \mathrm{XY} / 46, \mathrm{XY}, \mathrm{r}(21) / 45, \mathrm{XY},-21$ & ns & $\begin{array}{l}95 \% / 3 \% \mathrm{BL} \\
7 \% / 0 \% \text { sperm }\end{array}$ & infertility, pre-ICSI testing \\
\hline Total & 2 males 8 females & & & \\
\hline \multicolumn{5}{|l|}{ Balanced rearrangements } \\
\hline \multicolumn{5}{|l|}{ N/inv } \\
\hline $\begin{array}{l}\text { Gekas et al., } \\
2001[96]\end{array}$ & $46, X Y / 46, X Y, \operatorname{inv}(10)(p 11 q 21)$ & ns & $39 \%$ & sterility, candidate for ICSI \\
\hline $\begin{array}{l}\text { Kleszkowska et al., } \\
1990 \text { [21] (case 4) }\end{array}$ & $46, X X / 46, X X, \operatorname{inv}(12)(q 12 q 24)$ & $25 \mathrm{yr}$ & $90 \%$ & two SA \\
\hline $\begin{array}{l}\text { De la Fuente-Cortes } \\
\text { et al., } 2009 \text { [15] }\end{array}$ & $46, X Y / 46, X Y, \operatorname{inv}(14 q)$ & ns & $6 \% \mathrm{BL}$ & repeated miscarriages \\
\hline \multicolumn{5}{|l|}{$N / t$ balanced } \\
\hline $\begin{array}{l}\text { Stenchever } \\
\text { et al., } 1977 \text { [97] }\end{array}$ & $46, X X / 46, X X . t(1 ; 2)$ & $27 \mathrm{yr}$ & $50 \% \mathrm{BL}$ & habitual abortion \\
\hline $\begin{array}{l}\text { Stenchever } \\
\text { et al., } 1977 \text { [97] }\end{array}$ & $46, X X / 46, X X, t(1 ; 16)$ & $23 \mathrm{yr}$ & $50 \%$ BL & habitual abortion \\
\hline $\begin{array}{l}\text { Northup et al., } \\
2007 \text { [98] }\end{array}$ & $\begin{array}{l}46, X X / 46, X X, p s u \operatorname{dic}(1 ; 19) \\
\text { (q10;q13.42) }\end{array}$ & $27 \mathrm{yr}$ & $10 \% \mathrm{BL}, 0 \% \mathrm{SF}$ & premature ovarian failure \\
\hline $\begin{array}{l}\text { De la Fuente-Cortes } \\
\text { et al., } 2009 \text { [15] }\end{array}$ & $46, X X / 46, X X, t(1 p ; 21 q)$ & ns & $2 \% \mathrm{BL}$ & repeated miscarriage \\
\hline $\begin{array}{l}\text { Almeida et al., } \\
2012 \text { [99] }\end{array}$ & $46, X Y / 46, X Y, t(2 ; 2)(p 23 ; q 21.2)$ & $31 \mathrm{yr}$ & $\begin{array}{l}100 \% \text { BL, } 84 \% \\
\text { sperm }\end{array}$ & infertility, one SA \\
\hline $\begin{array}{l}\text { Lebbar et al., } 2008 \\
\text { [100] (patient 2) }\end{array}$ & $46, X Y / 46, X Y, t(2 ; 4 ; 12)$ & $40 \mathrm{yr}$ & $30 \% \mathrm{BL}$ & $\begin{array}{l}\text { secondary infertility, severe oligoasthenospermia, } \\
\text { necrospermia, leucospermia, teratospermia, one } \\
\text { child (not tested) }\end{array}$ \\
\hline Stenchever & $46, X X / 46, X X, t(2 ; 7)$ & $19 \mathrm{yr}$ & $75 \%$ & habitual abortion \\
\hline
\end{tabular}


Table 4 Somatic mosaicism for non centromeric rearrangement in asymptomatic carriers with poor reproductive history (Continued)

\begin{tabular}{|c|c|c|c|c|}
\hline $\begin{array}{l}\text { Stenchever } \\
\text { et al., } 1977 \text { [97] }\end{array}$ & $46, X X / 46, X X, t(2 ; 8)$ & $24 \mathrm{yr}$ & $50 \%$ & habitual abortion \\
\hline $\begin{array}{l}\text { Shaham et al., } \\
1992[101]\end{array}$ & $46, X X / 46, X X, t(2 ; 16)(p 23 ; q 24)$ & ns & ns & recurrent SA \\
\hline $\begin{array}{l}\text { Cantu and Ruiz, } \\
1986[102]\end{array}$ & $46, X Y / 46, X Y, t(3 ; 4)(q 22 ; q 35)$ & ns & ns & recurrent SA \\
\hline $\begin{array}{l}\text { Farrell, } 1991 \\
\text { [82] (case 1) }\end{array}$ & $46, X X / 46, X X, t(3 ; 6)(q 13.2 ; q 25.3)$ & ns & $9 \%$ & recurrent SA \\
\hline $\begin{array}{l}\text { Sciorra et al., } \\
1985 \text { [103] }\end{array}$ & $\begin{array}{l}46, X X / 46, X X, t(4 ; 5)(4 p t e r-> \\
4 q 21:: 5 q 32->5 q \text { ter;5pter-> } \\
5 q 34: 4 q 21->4 q \text { ter })\end{array}$ & ns & $\begin{array}{l}17-24 \% \mathrm{BL}, \\
0 \% \mathrm{SF}\end{array}$ & infertility, one miscarriage \\
\hline $\begin{array}{l}\text { De la Fuente-Cortes } \\
\text { et al., } 2009 \text { [15] }\end{array}$ & $46, X Y / 46, X Y, t(4 q ; 9 q)$ & ns & $2 \% \mathrm{BL}$ & recurrent SA \\
\hline $\begin{array}{l}\text { Meza-Espinoza } \\
\text { et al., } 2008 \text { [57] }\end{array}$ & $46, X Y / 46, X Y, t(5 ; 16 ; 17)$ & ns & $16 \% B L$ & habitual abortion \\
\hline $\begin{array}{l}\text { Kleszkowska et al., } \\
1990[21] \text { (case 2) }\end{array}$ & 46,XY/46,XY,rcp(9;13)(p21;q13) & $29 \mathrm{yr}$ & $70 \%$ & repeated miscarriage \\
\hline $\begin{array}{l}\text { Tuerlings et al., } \\
1998 \text { [104] }\end{array}$ & $46, X Y / 46, X Y, t(9 ; 20)(p 22 ; p 13)$ & ns & ns & two SA at 1st trimester \\
\hline $\begin{array}{l}\text { Lebbar et al., } 2008 \\
\text { [100] (patient 1) }\end{array}$ & $\begin{array}{l}46, X Y / 46, X Y, t(12 ; 14 ; 12 ; 9) \\
(q 13 ; q 32 ; p 13 ; q 32)\end{array}$ & $52 \mathrm{yr}$ & $20 \% B L$ & $\begin{array}{l}\text { secondary infertility, variable moderate } \\
\text { oligospermia, two healthy children }\end{array}$ \\
\hline $\begin{array}{l}\text { Gekas et al., } \\
\text { 2001] [96] }\end{array}$ & $46, X Y / 46, X Y, t(10 ; 13)(p 13.2 ; q 21)$ & ns & $19 \% B L$ & sterility, candidate for ICSI \\
\hline $\begin{array}{l}\text { Gekas et al., } \\
2001 \text { [96] }\end{array}$ & 46,XY/46/XY,t(11;19)(p11.2;q12) & ns & $79 \%$ BL & sterility, candidate for ICSI \\
\hline $\begin{array}{l}\text { Clementini et al., } \\
2005 \text { [105] }\end{array}$ & $46, X Y, / 46, X Y, t(15 ; 20)$ & ns & $3 \% \mathrm{BL}$ & sterility, candidate for ICSI \\
\hline Total & 13 males 10 females & & & \\
\hline
\end{tabular}

with reproductive failure was a carrier of mosaicism for unbalanced Rea.

The data on the incidence of mosaicism for balanced Reas obtained from the analysis of studies on patients with reproductive failures are consistent with corresponding data from a report on a constitutional chromosome analysis in 74,306 consecutive patients [21]. They reported an incidence of N/rcp carriers among all reciprocal translocation carriers as 1: 120 and incidence of N/inv carriers among all inversion carriers as $1: 25$. Corresponding figures from the present study are 1: 65 and 1: 34 .

It was noted above that two groups of patients with reproductive problems, i.e. patients with infertility and

Table 5 Somatic mosaicism for non centromeric rearrangementin asymptomatic carriers, fortitous findings

\begin{tabular}{|c|c|c|c|c|}
\hline Reference & Karyotype & $\begin{array}{l}\text { Age at } \\
\text { ascertainment }\end{array}$ & $\begin{array}{l}\text { Proportion of } \\
\text { abnormal cell line(s) }\end{array}$ & Indication for testing \\
\hline \multicolumn{5}{|l|}{ Balanced rearrangements } \\
\hline $\begin{array}{l}\text { Kleszkowska et al., } \\
1990 \text { [21] (case 1) }\end{array}$ & 46,XY/46,XY,rсp(1;9)(p13.1;p12.2) & $27 \mathrm{yr}$ & $50 \% \mathrm{BL}$ & fortitous finding \\
\hline $\begin{array}{l}\text { Schmid, Hatfield, } \\
1962[106]\end{array}$ & $\begin{array}{l}46, X X / 46, X X, \tan (2 ; 13,14, o r 15)(p 11.2 ; \\
q 26,32, o r 34)\end{array}$ & $86 \mathrm{yr}$ & $25 \% \mathrm{BL}$ & $\begin{array}{l}\text { a child and a grandchild } \\
\text { with a different Rea }\end{array}$ \\
\hline Leegte et al., 1998 [83] & $46, X X / 46, X X, t(3 ; 7)(q 26.2 ; p 14)$ & 64 yo & $40 \%$ BL & $\begin{array}{l}46, X Y \text { son with two stillborn } \\
\text { children }\end{array}$ \\
\hline $\begin{array}{l}\text { de Pina Neto, Ferrari, } \\
1980 \text { [107] }\end{array}$ & $46, X X / 46, X X, t(3 ; 20)$ de novo & $6 \mathrm{yr}$ & $54 \% \mathrm{BL}$ & $\begin{array}{l}\text { a sibs with a different } \\
\text { maternal Rea }\end{array}$ \\
\hline $\begin{array}{l}\text { Couzin et al., } \\
1987[108]\end{array}$ & $46, X Y / 46, X Y, t(7 ; 14)(q 32 ; q 11)$ & adult & $8 \% B L$ & a child with trisomy 21 \\
\hline $\begin{array}{l}\text { Kleszkowska et al., } \\
1990 \text { [21] (case 3) }\end{array}$ & 46,XX/46,XX,ins(14;13)(q24.1;q31.1q32.3 & $24 \mathrm{yr}$ & $60 \% \mathrm{BL}$ & $\begin{array}{l}\text { trilogy of Fallot, } 46, X Y \text { child } \\
\text { with tetralogy Fallot }\end{array}$ \\
\hline Total & 2 males 4 females & & & \\
\hline
\end{tabular}


Table 6 Sex ratio in carriers of somatic/gonadal mosaicism for structural and autosomal mosaicism

\begin{tabular}{|c|c|c|c|c|}
\hline \multirow[t]{2}{*}{ Group } & \multicolumn{2}{|c|}{$\begin{array}{l}\text { Unbalanced } \\
\text { rearrangements }\end{array}$} & \multicolumn{2}{|c|}{$\begin{array}{l}\text { Balanced } \\
\text { rearrangements }\end{array}$} \\
\hline & Males & Females & Males & Females \\
\hline $\begin{array}{l}\text { Affected carriers of } \\
\text { gonadal mosaicism }\end{array}$ & 2 & 10 & & \\
\hline $\begin{array}{l}\text { Asymptomatic carriers } \\
\text { of gonadal mosaicism, } \\
\text { abnormal offspring }\end{array}$ & 6 & 27 & 5 & 4 \\
\hline $\begin{array}{l}\text { Asymptomatic carriers } \\
\text { of gonadal mosaicism, } \\
\text { unaffected offspring }\end{array}$ & 1 & 1 & 4 & 4 \\
\hline Subtotal, $n=64$ & 9 & 38 & 9 & 8 \\
\hline $\begin{array}{l}\text { Asymptomatic carriers } \\
\text { of somatic mosaicism, } \\
\text { poor reproductive history }\end{array}$ & 2 & 8 & 13 & 10 \\
\hline $\begin{array}{l}\text { Asymptomatic carriers } \\
\text { of somatic mosaicism, } \\
\text { fortotous findings }\end{array}$ & & & 2 & 4 \\
\hline Subtotal, n=39 & 2 & 8 & 15 & 14 \\
\hline Total, $n=103$ & 11 & 46 & 24 & 22 \\
\hline Sex ratio & $0.24 *$ & & 1.09 & \\
\hline
\end{tabular}

* different from population ratio of $1.06, p<0.0001$

patients with repeated miscarriage, differ by both rate of chromosome abnormalities and SR among carriers of chromosome abnormalities. In couples with repeated miscarriage there was a notable female predominance among carriers of reciprocal translocations (113 M/ $203 \mathrm{~F}, \mathrm{SR}=0.56)$ unlike a slight male predominance among infertile couples $(63 \mathrm{M} / 51 \mathrm{~F}, \mathrm{SR}=1.2)$. The rate of reciprocal translocations in infertile individuals is lower compared to the rate in patients with repeated miscarriage both for males $(0.53 \%$ vs $0.86 \%)$ and for females $(0.4 \%$ vs $1.54 \%)$.

Three carriers of mosaicism for non centromeric Rea were detected among infertile patients (two cases of $\mathrm{N}$ / rcp and one case of $\mathrm{N} / \mathrm{inv}$ ), for a rate of $0.1 \%$, while among patients with repeated miscarriage eight cases were detected (five $\mathrm{N} / \mathrm{rcp}$, two $\mathrm{N} / \mathrm{inv}$, and one $\mathrm{N} / \mathrm{del}$ ), for a rate of $0.28 \%$. These figures are consistent with the overall lower frequency of carriers of non mosaic chromosomal abnormality among infertile patients of $1.1 \%(328 / 30,376)$ compared to $1.9 \%(505 / 26,384)$ among patients with miscarriages.

Reviewing data from prenatal amniocentesis samples, Shapira et al. [22] reported the rates of mosaic balanced reciprocal translocations as $<0.02-0.1$ per 1,000 samples and suggested that these rates may approximate the true frequency in the general population. However, mosaicism detected in amniocytes might not be confirmed in blood cells postnatally. For example, in the collaborative study of Hsu et al., [23] 13 cases of mosaic balanced reciprocal translocations were identified in 179,663 amniocenteses. However, at birth, five cases were not followed up, five newborns did not have confirmed mosaicism, and in only three cases was mosaicism confirmed. This study also identified four cases of mosaic inversions, with two of the cases confirmed in the newborn infants (0.01 per 1000).

In addition, Hsu et al., [23] commented that when a mosaicism is diagnosed along with a $46, \mathrm{XX}$ cell line, the possibility of maternal cell contamination might be suspected. One more aspect should be taken into consideration, namely that maternal age distribution in couples referred to prenatal testing is different from that in the general population. Further studies are needed before making any conclusion about maternal age effect on formation of mosaicism for structural Reas.

With respect to mosaicism for unbalanced rearrangements, it should be noted that many of the prenatally detected carriers, being abnormal, undergo spontaneous abortion or termination. In the same study of Hsu et al. [23], 17 cases of mosaicism for deletion were detected. Four of them were terminated, three were abnormal at birth, three normal newborns were not followed up, five normal newborns did not have confirmed mosaicism, and two normal newborns had confirmed mosaicism. One of them, with a low-grade mosaicism ( $2 \%$ of abnormal cells in blood sample) was reported to be normal at 7 months. Of three cases prenatally diagnosed as carriers of mosaicism for ring chromosome, two were abnormal (aborted) and one was a normal infant with a low-grade (8\%) mosaicism.

As noted above, for determination of population rates of mosaicism for structural abnormalities, we chose to analyze combined data from studies of asymptomatic carriers with reproductive failure. Since reproductive failure affects about $15 \%$ of couples, one may calculate population rates of N/rcp, N/inv, N/unbal Rea as 0.02 $\%$, $0.005 \%$, and $0.002 \%$, respectively. Therefore, population rates for balanced Reas calculated in the present study, are consistent with figures from prenatal samples [22]. However, it should be noted that these figures are most probably underestimated since in many cases mosaicism goes undetected because of the presence of normal cell line.

It should be stressed that mosaicism confined to the germline is more difficult to detect, and recent evidence suggests that it may be far more widespread than previously assumed [24]. Therefore, we support the view of Shapira et al. [22] and many other researchers: even if mosaicism is not detected, genetic counseling for chromosomally normal parents, with a prior aneusomic offspring or fetal loss, should always address the theoretical possibility of recurrence in a future pregnancy resulting from gonadal mosaicism. 
Table 7 Autosome rearrangements in patients with reproductive failure

\begin{tabular}{|c|c|c|c|c|c|c|c|c|c|}
\hline \multirow[t]{2}{*}{ Groups } & \multicolumn{2}{|l|}{$\begin{array}{l}\text { No. of carriers of non } \\
\text { mosaic rearrangement }\end{array}$} & \multicolumn{3}{|c|}{$\begin{array}{l}\text { Balanced nonmosaic } \\
\text { rearrangements, } \mathrm{n}(\%)\end{array}$} & \multirow[t]{2}{*}{$\begin{array}{l}\text { Other non mosaic } \\
\text { rearrangements }\end{array}$} & \multicolumn{2}{|c|}{$\begin{array}{l}\text { Mosaicism for structural } \\
\text { rearrangement excluding SCM }\end{array}$} & \multirow[t]{2}{*}{ Source } \\
\hline & & & $\begin{array}{l}\text { Reciprocal } \\
\text { translocation }\end{array}$ & $\begin{array}{l}\text { Robertsonian } \\
\text { translocation or } \\
\text { isochromosome }\end{array}$ & Inversion & & $\begin{array}{l}\text { Non centromeric } \\
\text { rearrangement }\end{array}$ & $\begin{array}{l}\text { Centromeric } \\
\text { rearrangement }\end{array}$ & \\
\hline \multirow{3}{*}{$\begin{array}{l}\text { Couples with } \\
\text { infertility } \\
(n=27,168)\end{array}$} & Males $(n=13,573)$ & 156 & 63 & 70 & 20 & 1 & & \multirow[t]{3}{*}{2 (rob) } & \multirow{3}{*}{$\begin{array}{l}{[93,105} \\
109-117]\end{array}$} \\
\hline & Females $(n=13,595)$ & 111 & 51 & 22 & 36 & 2 & & & \\
\hline & Sex ratio & & 1.2 & 3.8 & 0.6 & & & & \\
\hline \multirow{2}{*}{$\begin{array}{l}\text { Patients with } \\
\text { infertility } \\
(n=3,208)\end{array}$} & Males $(n=2,196)$ & 44 & 20 & 18 & 3 & 1 & 3 (2 rcp, 1 inv) & & \multirow[t]{4}{*}{ [96] } \\
\hline & Females $(n=1,012)$ & 21 & 7 & 7 & 7 & & & & \\
\hline \multirow{2}{*}{$\begin{array}{l}\text { Patients and } \\
\text { couples with } \\
\text { infertility, } \\
\text { total } \\
(n=30,376)\end{array}$} & Males $(n=15,769)$ & $196(1.3 \%)$ & $83(0.53 \%)$ & $88(0.56 \%)$ & $23(0.15 \%)$ & 2 & \multirow[t]{2}{*}{3 (2 rсp, 1 inv) } & \multirow[t]{2}{*}{2 (rob) } & \\
\hline & Females $(n=14,607)$ & $132(0.9 \%)$ & $58(0.4 \%)$ & $29(0.2 \%)$ & $43(0.29 \%)$ & 2 & & & \\
\hline \multirow{3}{*}{$\begin{array}{l}\text { Couples with } \\
\text { repeated } \\
\text { miscarriages } \\
(n=26,384)\end{array}$} & Males $(n=13,192)$ & $187(1.5 \%)$ & $113(0.86 \%)$ & $54(0.41 \%)$ & 19 (0.14\%) & 1 & 5 ( 4 rcp, 1 inv) & & \multirow{4}{*}{$\begin{array}{l}{[15,18,57,89} \\
105,117-126\end{array}$} \\
\hline & Females $(n=13,192)$ & 318 (2.5\%) & $203(1.54 \%)$ & $94(0.74 \%)$ & $18(0.14 \%)$ & 3 & \multirow{2}{*}{\multicolumn{2}{|c|}{3 ( $1 \mathrm{rcp}, 1$ inv, 1 del) }} & \\
\hline & Sex ratio & & 0.56 & 0.56 & 1.1 & & & & \\
\hline $\begin{array}{l}\text { Combined } \\
\text { data }\end{array}$ & 56,760 & 833 & 439 & 262 & 101 & 8 & $\begin{array}{l}11 \text { ( } 7 \text { rcp, } 3 \\
\text { inv, } 1 \text { del) }\end{array}$ & 2 (rob) & \\
\hline
\end{tabular}




\section{Conclusions}

A high proportion of abnormal Rea cells ( $>50 \%$ ) detected in cultured T-lymphocytes is associated with clinical manifestation of chromosomal imbalance. A strong female prevalence among carriers of mosaicism for unbalanced Rea suggests male-specific selection against abnormal cells rather than impairment of male gametogenesis. The latter suggests a better prognosis for male fetuses. These findings should be taken into consideration when counseling patients referred after a diagnosis of mosaicism for unbalanced rearrangement in a fetus.

\section{Competing interests}

The authors declare that they have no competing interests.

\section{Authors' contributions}

N.V.K. and P.D.C. performed the literature search, analyzed the data and wrote the manuscript. The authors alone are responsible for the content and writing of the paper. Both authors read and approved the final manuscript.

\section{Author details}

'Department of Inherited Diseases, The Turner's Scientific and Research Orthopaedic Institute for Children, Parkovaya Str. 64-68, St. Petersburg 196603, Russian Federation. ${ }^{2}$ Department of Pediatrics, University of California San Francisco, San Francisco, CA 94143, USA. ${ }^{3}$ ResearchDx Inc., Irvine, CA 92618, USA.

Received: 26 August 2015 Accepted: 21 November 2015

\section{Published online: 28 January 2016}

\section{References}

1. Zlotogora J. Germ line mosaicism. Hum Genet. 1998;102:381-6.

2. Kovaleva NV. Sex-specific chromosome instability in early human development. Am J Med Genet. 2005;136A:401-13.

3. Cheung SW, Shaw CA, Scott DA, Patel A, Sahoo T, Bacino CA, et al. Microarray-based CGH detects chromosomal mosaicism not revealed by conventional cytogenetics. Am J Med Genet. 2007;143A:1679-86.

4. Pagon RA, Hall JG, Davenport SLH, Aase J, Norwood TH, Hoehn HW. Abnormal skin fibroblast cytogenetics in four dysmorphic patients with normal lymphocytes chromosomes. Am J Hum Genet. 1979;31:54-61.

5. Kovaleva NV. Nonmosaic balanced homologous translocations: some may be mosaic. Am J Med Genet. 2007;143A:2843-50.

6. Barber JCK. Directly transmitted unbalanced chromosome abnormalities and euchromatic variants. J Med Genet. 2005:42:609-29.

7. Gu W, Zhang F, Lupski JR. Mechanisms for human genomic rearrangements. PathoGenetics. 2008:1:4

8. Clarke CM, Edwards JH, Smallpiece V. 21 trisomy/normal mosaicism in an intelligent child with some mongoloid characteristics. Lancet. 1961;1:1028.

9. Ridler MA, Shapiro A, Delhanty JD, Smith GF. A mosaic mongol with normal leucocyte chromosomes. Br J Psychiatry. 1965;111:183-5.

10. Zellweger H, Abbo G, Nielsen MK, Wallwork K. Mosaic mongolism with normal chromosomal complement in the white blood. Humangenetik. 1966;2:323-7.

11. Biesecker $L G$, Spinner NB. A genomic view of mosaicism and human disease. Nat Rev Genet. 2013:14:307-20.

12. Boué $A$, Gallano P. Collaborative study of the segregation of inherited structural rearrangements in 1,356 prenatal diagnoses. Prenat Diagn. 1984;4: 45-67.

13. Daniel A, Hook E, Wulf G. Collaborative USA data on prenatal diagnosis for parental carriers of chromosome rearrangements: risks of unbalanced progeny. In: Daniel A, editor. The cytogenetics of Mammalian autosomal rearrangements. New York, USA: Alan R Liss; 1988. p. 73-162.

14. Faraut T, Mermet M-A, Demongeot J, Cohen O. Cooperation of selection and meiotic mechanisms in the production of imbalances in reciprocal translocations. Cytogenet Cell Genet. 2000;88:15-21.

15. De la Fuente-Cortés BE, Cerda-Flores RM, Dávila-Rodríguez MI, García-Vielma C, De la Rosa Alvarado RM, Cortés-Gutiérrez El. Chromosomal abnormalities and polymorphic variants in couples with repeated miscarriage in Mexico. Reprod Biomed Online. 2009;18:543-8.

16. Chandley AC, Edmond P, Christie S, Gowans L, Fletcher O, Frackievicz A, et al. Cytogenetics and infertility in man. I. Karyotype and seminal analysis: results of a 5-year survey of men attending a subfertility clinic. Ann Hum Genet. 1975;39:231-54.

17. Lippman-Hand A, Vekemans M. Balanced translocations among couples with two or more spontaneous abortions: are males and females equally likely to be carriers? Hum Genet. 1983;63:252-7.

18. Al Hussain MAl, Al-Nuaim L, Abu Talib Z, Zaki OK. Cytogenetic study in cases with recurrent abortion in Saudi Arabia. Ann Saudi Med. 2000;20:2336.

19. Pergament $E$, Fiddler $M$, Cho N. Sexual differentiation and preimplantation cell growth. Hum Reprod. 1994;9:1730-2.

20. Alfarawati S, Fraguoli E, Colls P, Stevens J, Gutiérrez-Mateo C, Schoolcraft WB, et al. The relationship between blastocyst morphology, chromosomal abnormality, and embryo gender. Fertil Steril. 2011:95:520-4.

21. Kleczkowska A, Fryns JP, Van den Berghe H. On the variable effect of mosaic normal/balanced chromosomal rearrangements in man. J Med Genet. 1990; 27:505-7.

22. Shapira SK, Orr-Urtreger A, Shaffer LG. Constitutional mosaicism for a chromosome 9 inversion resulting in recombinant aneusomy in an offspring. Am J Med Genet. 1997:69:360-4.

23. Hsu LY, Yu MT, Richkind KE, Van Dyke DL, Crandall BF, Saxe DF, et al. Incidence and significance of chromosome mosaicism involving an autosomal structural abnormality diagnosed prenatally through amniocentesis: a collaborative study. Prenat Diagn. 1996:16:1-28.

24. Delhanty JDA. Inherited aneuploidy: germline mosaicism. Cytogenet Genome Res. 2011;133:136-40.

25. Freitas ÉL, Gribble SM, Simioni M, Vieira TP, Prigmore E, Krepischi AC, et al. A familial case with interstitial 2 q36 deletion: variable phenotypic expression in full and mosaic state. Eur J Med Genet. 2012;55:660-5.

26. Naritomi K, Hirayama K. Partial trisomy of distal $8 \mathrm{q}$ derived from mother with mosaic 8q23.3-> 24.13 deletion, and relatively mild expression of trochorhinophalangeal syndrome I. Hum Genet. 1989;82:199-201.

27. Magenis RE, Lahr M, Hefits-Borchardt VA, Lawce H, Wilson T. Inherited microdeletion syndrome, due to loss of single band 9q31.3: the continuing case for high resolution chromosome studies in unexplained mild mental retardation. Am J Hum Genet. 1989;45(suppl):A81.

28. Zori RT, Lupski JR, Heju Z, Greenberg F, Killian JM, Gray BA, et al. Clinical, cytogenetic, and molecular evidence for an infant with Smith-Magenis syndrome born from a mother having a mosaic 17p11.2p12 deletion. Am J Med Genet. 1993:47:504-11.

29. Cox H, Stewart H, Hall L, Donnai D. Phenotypic spectrum of interstitial 7p duplication in mosaic and non-mosaic forms. Am J Med Genet. 2002:109: 306-10.

30. Kennedy SJ, Teebi AS, Adatia I, Teshima I. Inherited duplication, dup(8)(p23. $1 \mathrm{p} 23.1)$ pat, in a father and daughter with congenital heart defects. Am J Med Genet. 2000;104:79-80.

31. Pfeiffer RA, Schütz C. Tandem duplication 11q23-ter in the dysmorphic child of a retarded mother mosaic for the same anomaly with no apparent abnormalities. Ann Genet. 1993;36:163-6.

32. Barber JCK, Zhang S, Friend N, Collins AL, Maloney VK, Hastings R, et al. Transmitted duplications of proximal $16 \mathrm{q}$ flanked by heterochromatin are not euchromatic variants and show no evidence of heterochromatic position effect. Cytogenet Genome Res. 2006;114:351-8.

33. Moog U, Engelen JJM, de Die-Smulders CEM, Albrechts JCM, Loneus WH, Haagen AAM, et al. Partial trisomy od the short arm of chromosome 18 due to inversion duplication and direct duplication. Clin Genet. 1994;46:423-9.

34. Fryns JP, Van den Berghe $H$. Ring chromosome 22 in a mentally retarded child and mosaic 45, XX,-15,-22,+t(15;22)(p11;q11)/46, XX, r(22)/46, XX karyotype in the mother. Hum Genet. 1979;47:213-6.

35. De Pater JM, Smeeth DFC, Scheres JMJC. Unique mosaicism of structural chromosomal rearrangement: Is chromosome 18 preferentially involved? Am J Med Genet. 2003;119A:26-31

36. Galjaard R-J H, van der Linde HC, Eussen BHJ, de Vries BBA, Wounters $\mathrm{CH}_{\text {, }}$ Oostra BA, et al. Isolated posaxial polydactyly type B with mosaicism of a submicroscopic unbalanced translocation leading to an extended phenotype in offspring. Am J Med Genet. 2003;121A:168-73.

37. Galán-Gómez E, Sutphen R, Ranells J, Kousseff BG. Reproduction in a mosaic cri-du-chat patient. Am J Hum Genet. 1994;55 Suppl 3:A279. 
38. Johnson El, Marinescu RC, Punnett HH, Tenenholz B, Overhauser J. $5 p 14 d e l e t i o n$ associated with microcephaly and seizures. J Med Genet. 2000;37:125-7.

39. McDonald DM, Emanul BS, Driscoll DA, Eunpu DL, Zackai EH. Chromosomal aneuploidy masquerading as autosomal dominant inheritance. Am J Hum Genet. 1988:43 suppl 3:A169.

40. Niebuhr E. Cytologic observations in 35 individuals with a $5 p$ - karyotype. Hum Genet. 1978:42:143-56.

41. Van Tuinen P, Grignon J, Miller P, Schalk P, Emery M, Brooks C, et al. Serendipitous detection of low-level somatic mosaicism in the mother of a child with deletion 5 p14.2: limitation of "limited studies" in relatives of cytogenetically abnormal individuals. Am J Hum Genet. 2001;69 Suppl 4:851.

42. Brandriff B, Gordon LA, Crawford BB, Schonberg SA, Golabi M, Charzan S, et al. Sperm chromosome analysis to assess potential germ cell mosaicism. Clin Genet. 1988:34:85-9.

43. Michalová K, Klouček F, Musolová J. Deletion of $13 q$ in two patients with retinoblastoma, one probably due to $13 q$ - mosaicism in the mother. Hum Genet. 1982;61:264-6.

44. Kokkonen $\mathrm{H}$, Leisti J. An unexpected recurrence of Angelman syndrome suggestive of maternal germ-line mosaicism of del(15)(q11q13) in a Finnish family. Hum Genet. 2000:107:83-5.

45. Rump P, Dijkhuizen T, Sikkema-Raddatz B, Lemmink HH, Vos YJ, Verheij JBGM, et al. Drayer's syndrome of mental retardation, microcephaly, short stature and absent phalanges is caused by a recurrent deletion of chromosome 15(q26.2- > qter). Clin Genet. 2008;74:455-62.

46. Sánchez J, Fernández R, Madruga M, Bernabeu-Wittel J, Antiñolo G, Borrego S. Somatic and germ-line mosaicism of deletion 15q11.2-q13 in a mother of dyzigotic twins with Angelman syndrome. Am J Med Genet. 2014;164A: 370-6.

47. Hoo JJ, Lowry RB, Lin CC, Haslam RHA. Recurrent de novo interstitial deletion of 16 q in two mentally retarded sisters. Clin Genet. 1985;27:420-5.

48. Garcia-Heras J, Kilani RA, Martin RA, Lamp S. A deletion of proximal 20p inherited from a normal mosaic carrier mother in a newborn with panhypopituitarism and craniofacial dysmorphism. Clin Dysmorphol. 2005: 14:137-40

49. Eussen $B H$, van de Laar I, Douben $H$, van Kempen $L$, Hochstenbach $R$, De Man SA, et al. A familial inverted duplication 2q33-q34 identified and delineated by multiple cytogenetic techniques. Eur J Med Genet. 2007;50:112-9.

50. Bernardini L, Sinibaldi L, Ceccarini C, Novelli A, Dallapiccola B. Reproductive history of a healthy woman with mosaicduplication of chromosome $4 \mathrm{p}$. Prenat Diagn. 2005;25:283-5.

51. Tosca L, Brisset S, Petit FM, Lecerf L, Rousseau G, Bas C, et al. Recurrent 70.8 Mb 4q22.2q32.3 duplication due to ovarian germinal mosaicism. Eur J Hum Genet. 2010;18:882-8.

52. Fan YS, Siu VM, Jung JH, Farrell SA, Côté GB. Direct duplication of 8p21.3-> p23.1: a cytogenetic anomaly associated with developmental delay without consistent clinical features. Am J Med Genet. 2001;103:231-4.

53. Tonk V, Schneider NR, Delgado MR, Mao J, Schultz RA. Identification and molecular confirmation of a small chromosome 10q duplication [dir dup(10)(q24.2-> q24.3)] inherited from a mother mosaic for the abnormality. Am J Med Genet. 1996;61:16-20.

54. Hocking T, Thompson E, Bain S, Barrat W, Digenis M. Mosaic structural abnormalities - are rare? Bull Hum Genet Soc Australasia. 1999;12:A28.

55. Babovic-Vuksanovic D, Westman JA, Jalal SM, Lindor NM. Clinical characteristics associated with dup17(q24q25.1) in a mosaic mother and two non-mosaic daughters. Clin Dysmorphol. 1998;7:171-6.

56. Flowers N, Kelley J, Sigurjonsson S, Bruno DL, Pertile MD. Maternal mosaicism for a large segmental duplication of $18 \mathrm{q}$ as a secondary finding following non-invasive prenatal testing and implications for test accuracy. Prenat Diagn. 2015;35:1-4.

57. Meza-Espinoza JP, Anguiano LO, Rivera H. Chromosomal abnormalities in couples with reproductive disorders. Gynecol Obstet Invest. 2008;66:237-40.

58. Fryns JP, Kleczkowska A, Smeets $E$, van den Berghe $H$. Transmission of ring chromosome 18 46, XX/46, XX, r(18) mosaicism in a mother and ring chromosome 18 in her son. Ann Genet. 1992;35:121-3.

59. Flejter WL, Finlinson D, Root S, Nguen W, Brothman AR, Viskochil D. Familia ring (19) mosaicism: case report and review. Am J Med Genet. 1996;66:276-80.

60. Phelan MC. Deletion 22q13.3 syndrome. Orphanet J Rare Dis. 2008;3:14.

61. Engel U, Bohlander SK, Bink K, Hinney B, Laccone F, Bartels I. Pseudo dicentric chromosome $(5 ; 21)$ : a rare example of maternal germline mosaicism. Hum Reprod. 2001;16:63-6.
62. Kouru KH, Malmgren $\mathrm{H}$, White I, Blennow E. Hidden mosaicism for a structural chromosome rearrangement: a rare explanation for recurrent miscarriages and affected offspring? Fertil Steril. 2011;95:806-8.

63. Gijsbers ACJ, Dauwerse JG, Bosch CAJ, Boon EMJ, van den Ende W, Kant SG, et al. Three new cases with mosaicism involving a normal cell line and a cryptic unbalanced autosomal reciprocal translocation. Eur J Med Genet. 2011;54:e409-12.

64. Papenhausen PR, Mueller OT, Kouseff BG, Tedesco TA. Partial trisomy 21 (q22.1->qter) due to a duplication on the short arm inherited from an asymptomatic mother. Amer J Hum Genet. 1991;49(suppl):271.

65. Al AS. Familial chromosomal abnormalities in ten families. Am J Hum Genet. 1998;64 Suppl 4:A126.

66. Eckel H, Wimmer $\mathrm{R}$, Volleth $\mathrm{M}$, Jakubiczka $\mathrm{S}$, Muschke $\mathrm{P}$, Wieacker $\mathrm{P}$. Intrachromosomal triplication 12p11.22-p12.3 and gonadal mosaicism of partial tetrasomy 12p. Am J Med Genet. 2006;140 A:1219-22.

67. Masada CT, Olney AH, Fordyce R, Sanger WG. Partial deletion of $14 q$ and partial duplication of $14 \mathrm{q}$ in sibs: testicular mosaicism for $\mathrm{t}(14 \mathrm{q} ; 14 \mathrm{q})$ as a common mechanism. Am J Med Genet. 1989;34:528-34.

68. Insley J, McDermott A, Parrington J. Familial structural chromosome abnormality with maternal mosaicism. Ann Genet. 1968;11:138-44.

69. D'Angelo CS, de Oliveira MA, de Castro CIE, Koiffmann CP. Molecular cytogenetic characterization of an inherited maternal duplication 20p11.21p13 associated with a small 20p11.21 deletion. Am J Med Genet. 2010;152A:3197-202

70. Wang BT, Hemmat M, Jayakar P, Boyar F, Chan P, El Naggar M, et al Paternal mosaic inv(20) resulting in a recombinant chromosome 20 in two siblings. Pediatr Int. 2010;52:492-5.

71. Aurias A, Prieur M, Dutrillaux B, Lejeune J. Systematic analysis of 95 reciprocal translocations of autosomes. Hum Genet. 1978:45:259-82.

72. Becker $\mathrm{KL}$, Albert A. Familial translocation mongolism: a carrier exhibiting nonacrocentric translocation. Proc Mayo Clin. 1963:38:261.

73. Gardner RJ, Dockery HE, Fitzgerald PH, Parfitt RG, Romain DR, Scobie N, et al. Mosaicism with a normal cell line and an autosomal structural rearrangement. J Med Genet. 1994;31:108-14.

74. Simonova W, Kuzmicheva IA, Temnikova El, Mikhailov VM. A particular clinical case of cri-du-chat syndrome due to paternal balanced translocation. Med Genet (Rus). 2005:4(Suppl):266-7.

75. Sciorra LJ, Schlenker E, Toke D, Brady-Yasbin S, Day-Salvatore D, Lee ML. Low level mosaicism for a balanced 7;14 translocation in the father of an abnormal 7q + child. Am J Med Genet. 1992;42:296-7.

76. Opheim KE, Brittingham A, Chapman D, Norwood TH. Balanced reciprocal translocation mosaicism: how frequent? Am J Med Genet. 1995;57:601-4.

77. Yatsenko SA, del Valle TM, Fernandes PH, Wiszniewska J, Gallego M, Herrera $J$, et al. Molecular characterization of a balanced rearrangement of chromosome 12 in two siblings with Noonan syndrome. Am J Med Genet A. 2009:149A:2723-30.

78. Tinkel-Vernon H, Finkernagel S, Desposito F, Pittore C, Reynolds K, Sciorra L. Patient with a deletion of chromosome $21 \mathrm{q}$ and minimal phenotype. Am J Med Genet. 2003;120A:142-3.

79. Mazzaschi RL, Love DR, Hayes I, George A. Inheritance of a ring chromosome 21 in a couple undergoing in vitro fertilization (IVF): a case report. Case Rep Genet. 2011;2011:158086.

80. Zackowski JL, Newlin A, Leichtman LG, Neu RL. Pericentric inversion of chromosome 1 inherited from a mosaic father. Cytogenet Cell Genet. 1995; 69(1-2):117A

81. Heil M, Ebrahim SAD, Van Dyke DL, Micale MA, Kurczynski TW, Yaron Y, et al Mosaicism for a balanced structural chromsomal rearrangement. Am J Hum Genet. 1997;61 Suppl 4:A127.

82. Farrel SA. Balanced reciprocal translocation mosaicism: new cases and a literature review. Am J Med Genet. 1991:40:345-7.

83. Leegte B, Sikkema-Raddatz B, Hordijk HR, Bouman K, van Essen T, Castedo S, et al. Three cases of mosaicism for balanced reciprocal translocations. Am J Med Genet. 1998:79:362-5.

84. Dupont C, Delahaye A, Burglen L, Tabet AC, Aboura A, Kanafani S, et al. First cryptic balanced reciprocal translocation mosaicism and familial transmission. Am J Med Genet. 2008;146A:2971-4.

85. Storto PD, Diehn TN, O'Malley DP, Bullard BA, Netzloff ML, VanDyke DL, et al. Satellited chromosome 10 detected prenatally in a fetus and confirmed as mosaic in a parent. Prenat Diagn. 1999;19:1088-9.

86. D'Alessandro E, Santiemma V, Lo Re ML, Ligas C, Del Porto G. $6 p$ deletion mosaicism in a woman with recurrent abortions and idiopathic hypoprolactiemia. Am J Med Genet. 1992;44:220-2. 
87. Reddy KS. A paternally inherited terminal deletion, del(8)(p23.1)pat, detected prenatally in an amniotic fluid sample: a review of deletion 8p23.1 cases. Prenat Diagn. 1999;9:868-72.

88. Kleczkowska A, Fryns J-P. Mosaic normal/unbalanced karyotype and recurrent fetal wastage. Am J Med Genet. 1990;36:379.

89. Dutta UR, Rajitha P, Pidugu VK, Dalal AB. Cytogenetic abnormalities in 1162 couples with recurrent miscarriages in southern region of India: report and review. J Assist Reprod Genet. 2011;28:145-9.

90. Sachs ES, Jahoda MG, Van Hemel JO, Hoogeboom AJ, Sandkuyl LA. Chromosome studies of 500 couples with two or more abortions. Obstet Gynecol. 1985;65:375.

91. Somprasit C, Aguinaga M, Cisneros PL, Torsky S, Carson SA, Buster JE, et al. Paternal gonadal mosaicism detected in a couple with recurrent abortions undergoing PGD: FISH analysis of sperm nuclei proves valuable. Reprod Biomed Online. 2004:9:225-30.

92. Lee MH, Park SY, Kim YM, Kim JM, Yoo KJ, Lee HH, et al. Molecular cytogenetic characterization of ring chromosome 4 in a female having a chromosomally normal child. Cytogenet Genome Res. 2005;111:175-8.

93. Scholtes MCW, Behrend C, Dietzel-Dahmen J, van Hoogstraten DG, Marx K, Wohlers $\mathrm{S}$, et al. Chromosomal aberrations in couples undergoing intracytoplasmic sperm injection: influence on implantation and ongoing pregnancy rate. Fertil Steril. 1998;70:933-7.

94. Tarlatzis BC, Toncheva DI, Vatev IT. Significance of chromosomal aberrations for the unsuccessful procedures of assisted reproduction. Eur J Obstet Gynecol Reprod Biol. 2000;88:181-7.

95. Hammoud I, Gomes DM, Bergere M, Wainer R, Selva J, Vialard F. Sperm chromosome analysis of an infertile patient with a 95\% mosaic r(21) karyotype and normal phenotype. Fertil Steril. 2009;91:930. e13-5.

96. Gekas J, Thepot F, Turleau C, Siffroi JP, Dadoune JP, Wasels R, et al. Chromosomal factors of infertility in candidate couples for ICSI: an equal risk of constitutional aberrations in women and men. Hum Reprod. 2001;16:82-90.

97. Stenchever MA, Parks KJ, Daines TL, Allen MA, Stenchever MR. Cytogenetics of habitual abortion and other reproductive wastage. Am J Obstet Gynecol. 1977;127:143-50.

98. Northup J, Griffis K, Hawkins J, Lockhart L, Velagaleti G. Unusual pseudo dicentric, psu dic(1;19)(q10;q13.42), in a female with premature ovarian failure. Fertil Steril. 2007;87:697. e5-e8.

99. Almeida C, Dória S, Moreira M, Pinto J, Barros A. Normal sperm in a 2:2 homologous male translocation carrier. J Assist Reprod Genet. 2012;29:665-8.

100. Lebbar A, Callier P, Baverel F, Marle N, Patrat C, Le Tessier D, et al. Two cases of mosaicism for complex chromosome rearrangements associated with secondary infertility. Am J Med Genet. 2008;146A:2651-6.

101. Shaham M, Beachman S, Vogel J, McGivans M, Carnahan A, Chaban PA, et al. Mosaicism for balanced and unbalanced structural chromosome rearrangements. Am J Hum Genet. 1992;51(suppl):A296.

102. Cantú JM, Ruiz C. On a prezygotic origin of normal/balanced translocation mosaics. Ann Genet. 1986;29:221-2.

103. Sciorra LL, Lee ML, Cuccurullo G. Translocation mosaicism in a woman having multiple miscarriages. Am J Med Genet. 1985;22:615-7.

104. Tuerlings JH, de France HF, Hamers A, Hordijk R, Van Hemel JO, Hansson K, et al. Chromosome studies in 1792 males prior to intra-cytoplasmic sperm injection: the Dutch experience. Eur J Hum Genet. 1998;6:194-200.

105. Clementini E, Palka C, lezzi I, Stuppia L, Guanciali-Franchi P, Tiboni GM Prevalence of chromosomal abnormalities in 2078 infertile couples referred for assisted reproductive techniques. Hum Reprod. 2005;20:437-42.

106. Schmid W, Hatfield D. Normal karyotype/translocation mosaic. Cytogenetics. 1962;1:210-6.

107. De Pina Neto JM, Ferrari I. Partial 3p trisomy and different rearrangements involving chromosome 3 in the proposita's family. Am J Med Genet. 1980;5: 25-33.

108. Couzin DA, Watt JL, Stephen GS. Structural rearrangements in the parents of children with primary trisomy 21. J Med Genet. 1987;24:280-2.

109. Mau UA, Bäckert IT, Kaiser P, Kiesel L. Chromosomal findings in 150 couples referred for genetic counselling prior to intracytoplasmic sperm injection. Hum Reprod. 1997;12:930-7.

110. Peschka B, Leygraaf J, Van der Ven K, Montag M, Schartmann B, Schubert R, et al. Type and frequency of chromosome aberrations in 781 couples undergoing intracytoplasmic sperm injection. Hum Reprod. 1999;14:2257-63.

111. Kayed HF, Mansour RT, Aboulghar MA, Serour Gl, Amer AE, Abdrazik A. Screening for chromosomal abnormalities in 2650 infertile couples undergoing ICSI. Reprod Biomed Online. 2006;12:359-70.
112. Meschede D, Lemcke B, Exeler JR, De Geyter C, Behre HM, Nieschlag E, et al. Chromosome abnormalities in 447 couples undergoing intracytoplasmic sperm injection-prevalence, types, sex distribution and reproductive relevance. Hum Reprod. 1998;13:576-82.

113. Riccaboni A, Lalatta F, Caliari I, Bonetti S, Somigliana E, Ragni G. Genetic screening in 2,710 infertile candidate couples for assisted reproductive techniques: results of application of Italian guidelines for the appropriate use of genetic tests. Fertil Steril. 2008;89:800-8.

114. Rosenbusch B. Somatic chromosomal abnormalities in couples undergoing infertility treatment by intracytoplasmic sperm injection. J Genet. 2010;89: $105-8$.

115. Artini PG, Papini F, Ruggiero M, Bartalini G, De Leo V, Scaravelli G, et al. Genetic screening in Italian infertile couples undergoing intrauterine insemination and in vitro fertilization techniques: a multicentric study. Gynecol Endocrinol. 2011;27:453.

116. Tiboni GM, Verna I, Giampietro F, Leonzio E, Impicciatore GG. Cytogenetic findings and reproductive outcome of infertile couples referred to an assisted reproduction program. Gynecol Endocrinol. 2011;27:669-74.

117. Gada Saxena S, Desai K, Shawale L, Ranjan P, Saranath D. Chromosomal aberrations in 2000 couples of Indian ethnicity with reproductive failure. Reprod Biomed Online. 2012;25:209-18.

118. Osztovics MK, Tóth SP, Wessely JA. Cytogenetic investigations in 418 couples with recurrent fetal wastage. Ann Genet. 1982;25:232-6.

119. Schwartz S, Palmer CG. Chromosomal findings in 164 couples with repeated spontaneous abortions: with special consideration to prior reproductive history. Hum Genet. 1983;63:28-34

120. Fryns JP, Kleczkowska A, Kubien E, Petit P, Van den Berghe H. Cytogenetic survey in couples with recurrent fetal wastage. Hum Genet. 1984;65:336-54.

121. Cantú JM, Hernández A, Jiménez-Sáinz M, Moller M, Rolon A, González-Flores, et al. Chromosome aberrations in 334 individuals with various types of abortion (including144 couples). Rev Invest Clin (Mex). 1985;37:131-4. 457.

122. Bourrouillou G, Colombies P, Dastugue N. Chromosome studies in 2136 couples with spontaneous abortions. Hum Genet. 1986:74:399-401.

123. Fryns JP, Van Buggenhout G. Structural chromosome rearrangements in couples with recurrent fetal wastage. Eur J Obstet Gynecol Reprod Biol. 1998;81:171-6.

124. Dubey S, Chowdhury MR, Prahlad B, Kumar R, Mathur R, Hamilton S, et al. Cytogenetic causes for recurrent spontaneous abortions - an experience of 742 couples (1484 cases). Indian J Hum Genet. 2005;11:94-8.

125. Nazmy NA. Cytogenetic studies of couples with reproductive failure in Alexandria, Egypt. J Egypt Public Health Assoc. 2008;83:255-71.

126. Sheth FJ, Liehr T, Kumari P, Akinde R, Sheth HJ, Sheth JJ. Chromosomal abnormalities in couples with repeated fetal loss: An Indian retrospective study. Indian J Hum Genet. 2013;19:415-22.

\section{Submit your next manuscript to BioMed Central and we will help you at every step:}

- We accept pre-submission inquiries

- Our selector tool helps you to find the most relevant journal

- We provide round the clock customer support

- Convenient online submission

- Thorough peer review

- Inclusion in PubMed and all major indexing services

- Maximum visibility for your research

Submit your manuscript at www.biomedcentral.com/submit 